\title{
Isolated Dextrocardia with Situs Solitus - Dextroversion in a Ugandan Baby: A Case Report
}

\author{
Sanjanaa Srikant (iD \\ Darshit Dave $\mathbb{D}^{2}$ \\ Dhara Dave ${ }^{3}$ \\ 'Department of Medicine, Makerere \\ University College of Health Sciences, \\ Kampala City, Uganda; ${ }^{2}$ Department of \\ Paediatrics, Uganda Martyrs Hospital \\ Lubaga, Kampala City, Uganda; \\ ${ }^{3}$ Department of Medicine, Texas Tech \\ University Health Sciences Center, \\ Lubbock, TX, USA
}

Background: Dextrocardia is a rare congenital abnormality in the general population, with an estimated incidence of 1 in 12,000 live births. Isolated dextrocardia, the condition of right-sided heart with situs solitus is most commonly referred to as dextroversion.

Case Presentation: A 2-month-old Ugandan baby boy discovered to have isolated dextrocardia with situs solitus presented with fever, cough, and difficulty in breathing. Physical examination revealed moderate respiratory distress, bilateral basal fine crepitations, tachycardia with regular pulse, and heart sounds best heard on the right hemithorax, but no murmurs. Chest X-ray confirmed right-sided cardiac shadow with cardiac apex pointing to right. Echocardiography showed D-loop ventricular morphology with right cardiac axis and ventricular apex to right, plus tiny patent ductus arteriosus. Abdominal ultrasound showed normal situs solitus; however, the left kidney was noted in pelvic space.

Conclusion: Isolated dextrocardia with situs solitus (dextroversion) is a rare scenario. This case exhibits a learning point in the significance of routine medical examination of patients to identify anomalies and upon suspicion a thorough evaluation to prevent wrong diagnosis or poor prognosis due to delayed management.

Keywords: isolated dextrocardia, dextroversion, situs solitus, Uganda, congenital abnormality

\section{Introduction}

In normal anatomy, the morphologic right atrium and ventricle are to the right of the morphologic left atrium and ventricle. However, in congenital cardiac malposition, chamber location is variable in relation to its morphologic counterpart. Dextrocardia is malpositioning of the heart, with the cardiac apex in the right hemithorax.

Dextrocardia is a rare congenital abnormality in the general population, with an estimated incidence of 1 in 12,000 live births ${ }^{1}$ and may be associated with significant additional cardiac malformations. The male-to-female incidence is $1: 1$. Dextrocardia with a normal abdominal situs has a high incidence of associated congenital cardiac anomalies, on the other hand, dextrocardia with situs inversus is associated with a lower incidence of congenital heart disease, $0-10 \%{ }^{2}$

The concept of situs applies to the pattern of the viscera as a whole and to each asymmetric viscus including the heart itself. The arrangements of the position of the abdominal viscera in dextrocardia may be normal (situs solitus), reversed (situs inversus), and indeterminate (situs ambiguous or isomerism) in $32-35 \%, 35-39 \%$, and $26-28 \%$ of cases, respectively. ${ }^{1,3}$

However, the atrial situs always corresponds to the visceral situs. ${ }^{4}$
Correspondence: Darshit Dave Department of Paediatrics, Uganda Martyrs Hospital Lubaga, P.O.Box 14130 Kampala City, Uganda

Email davedarshit94@gmail.com 
Isolated dextrocardia with situs solitus consists of right-sided cardiac apex, the morphologic left atrium is to the left of the morphologic right atrium, the right lung has three lobes whereas the left lung has two lobes, and the larger lobe of the liver is on the right, with the stomach and spleen on the left. ${ }^{5}$

\section{Case Presentation}

We report an unusual case of a 2-month-old Ugandan baby boy found to have isolated dextrocardia, who presented with a one-week history of fever $\left(37.8^{\circ} \mathrm{C}\right)$, flu, and cough as well as 3 days of difficulty in breathing with pulse oximetry at $85 \%$ on room air.

The baby was seronegative for HIV and had no history of TB exposure. The infant was born by spontaneous vaginal delivery and immunization was up-to-date. There was no history of diabetes, cocaine use, or any other drug intake in his mother. This was the 4 th born of 4 children all from the same non-consanguineous parents, and all siblings well and healthy.

On examination, the infant was critically ill. He had no pallor, no jaundice, some dehydration, no cyanosis, no edema. He had moderate respiratory distress (subcostal recession, chest indrawing, tachypnea, and grunting) and bilateral basal fine crepitations. Cardiovascular system examination showed tachycardia at 148 beats/minute, but a regular and full volume pulse. There was cardiac dullness on his right side, and heart sounds 1 and 2 were heard, best on the right hemithorax with the apex beat best located in the 5th right intercostal space, and neither a gallop rhythm nor murmur. Abdominal examination revealed no palpable organomegaly but had dullness on percussion in the right upper quadrant and tympanic note over the left hypochondrium.

The infant was COVID negative, malaria slide showed no plasmodium parasites, he had a normal white blood cell count, hemoglobin but raised platelet count of 727,000 cells/microliter. The abdominal scan revealed a normal situs solitus however the left renal fossa was empty with the left kidney noted to be in the lower abdomen at the pelvic space, but normal in size, shape, and echogenicity.

A chest X-ray confirmed a right-sided cardiac shadow and the cardiac apex pointing to the right, but no cardiac silhouette enlargement. The mediastinum was central, liver shadow on the right consistent with situs solitus, and right hemidiaphragm presenting at a higher level compared to the left. Heterogeneous opacities in the upper and middle left lung zones and clear pleural spaces (Figure 1).

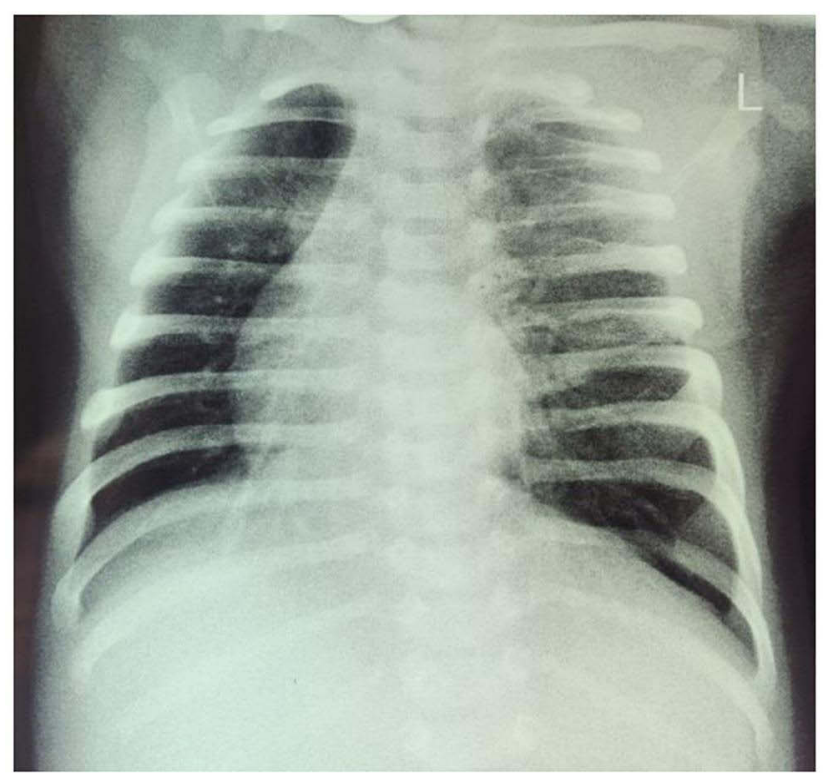

Figure I Chest $\mathrm{X}$-ray showing right-sided cardiac shadow with the apex pointing right and heterogeneous opacities in the left lung.

Echocardiography showed D-loop ventricular morphology with a right cardiac axis and the ventricular apex to the right. Also, a tiny patent ductus arteriosus with a moderate left to right shunting, and a stenotic superiorand inferior-vena caval inflow was noted at the right atrial junction, though no interventricular or interatrial septal defect. However, he had a normal visceral situs, and concordant atrio-ventricular-arterial (AVA) connections (Figure 2).

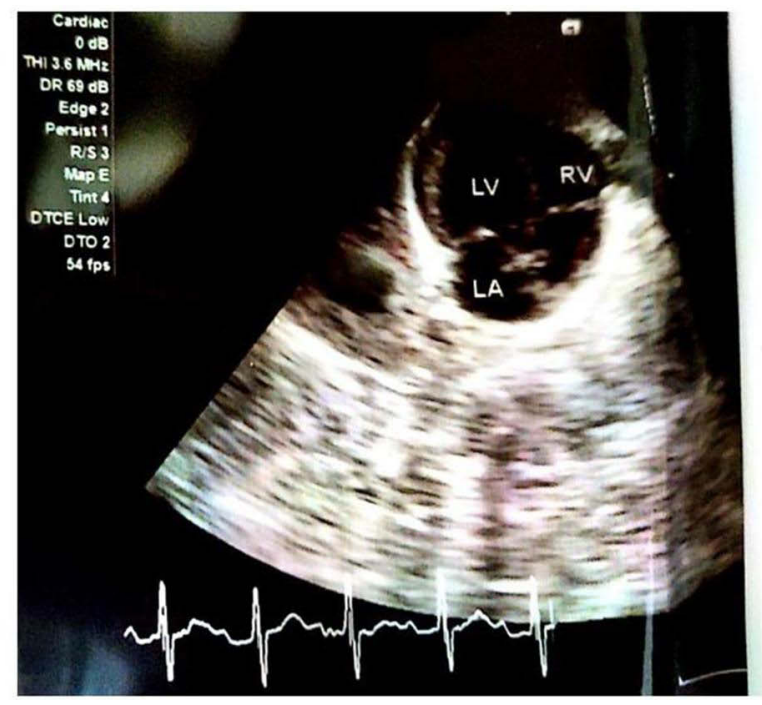

Figure 2 Cardiac echocardiography with D-loop ventricular morphology and right cardiac axis. 
The baby was managed on Oxygen therapy by low flow nasal prongs at 3 liters/minute, started on Intravenous (IV) Ceftriaxone-Sulbactam $384 \mathrm{mg}$ once daily, IV Amikacin $36 \mathrm{mg}$ once daily, IV furosemide $9 \mathrm{mg}$ stat dose then $5 \mathrm{mg}$ twice daily, Tablets Zinc $10 \mathrm{mg}$ once daily, IV Hydrocortisone $30 \mathrm{mg}$ thrice daily, and Nasogastric tube feeding.

\section{Discussion}

The human fetal heart develops from a primitive cardiac tube with sinus venosus, atrium, ventricle, bulbus cordis, and truncus arteriosus connected in series. Venous and arterial ends are fixed. The atria and venous return form simultaneously hence atria are fixed in position by the entering veins. The bulboventricular loop grows resulting in the bending of the cardiac tube forming morphologically right and left ventricles and thus it does not affect the atrial situs which therefore corresponds to the visceral situs. ${ }^{4,6}$

The direction of the initial formation of the cardiac loop determines the relative locations of the ventricles. The primitive heart tube may loop to the right (dextroor D-) forming a D-loop or to the left (levo- or L-) forming an L-loop. The bulbus cordis develops into the morphologic right ventricle and the ventricle of the bulboventricular loop develops into the morphologic left ventricle. The D-loop hence results in the morphologic right ventricle to the right of the morphologic left ventricle and thus is the normal (solitus) cardiac loop. ${ }^{7}$ Consequently, concordant cardiac loops are the D-loop with situs solitus and the L-loop with situs inversus, whereby concordance is relative to the type of visceroatrial situs. ${ }^{8}$

In the early stages of fetal life, with situs solitus and formation of the D-loop, the apex of the heart is in the right hemithorax. By the end of the first month of fetal life, the apex later migrates from the right thorax to the left hemithorax. Regardless of the atrial situs, all D-bulboventricular loops should accordingly end development with the heart in the left hemithorax (levocardia).

Dextrocardia can result from either a D-bulboventricular loop that fails to undergo a shift into the left hemithorax, or from an L-bulboventricular loop that completes its apical shift into the right hemithorax. However, isolated dextrocardia with situs solitus, D-loop ventricles, and concordant great arteries results from failure of the final leftward shift of ventricular apex during embryologic development with failure of rotation into the left hemithorax rather than an abnormal rotation of the apex into the right hemithorax. Regardless of the abnormal location of the ventricles, the atria and abdominal viscera maintain their situs solitus. ${ }^{9}$

Dextrocardia with situs solitus, D-loop ventricles, and normally related great arteries is termed dextroversion which results from failure of the final leftward shift of the ventricles during embryologic development. Although the morphologic right atrium and morphologic right ventricle remain to the right, they are located posterior to the corresponding left-sided chambers. This is the 2nd most common type of dextrocardia and has a $90 \%$ incidence of additional cardiac malformations. ${ }^{10,11}$

\section{Conclusion}

Though isolated dextrocardia is a benign condition, and in most cases, affected individuals can live a normal life without any symptoms or disability. However, it can often be associated with other congenital anomalies, which can lead to several problems such as failure to thrive, recurrent infections, and breathing difficulties in newborns and neonates.

Due to the possibility of other congenital anomalies associated with dextrocardia, diagnosing dextrocardia could be a bridge to the diagnosis of other cardiac anomalies. Doctors should encourage routine medical examination for their patients which could help identify this anomaly and upon suspicion, a thorough evaluation that involves imaging and genetic analysis is necessary to assess for other accompanying abnormalities, to prevent wrong diagnosis or death due to delayed management. Surgeons, radiologists, and radiographers should look out for this anomaly during the preoperative and surgical management of their patients.

Treatment of dextrocardia is symptomatic and supportive when needed. If the condition is associated with other more serious heart malformations, treatment will vary. Our patient's dextrocardia was not associated with serious heart malformations. He only had a tiny patent ductus arteriosus which did not require surgical intervention with immediate effect.

\section{Informed Consent/Ethical Approval}

Written informed consent was obtained from the legal guardian of the patient for publication of the medical history/course and this case report with accompanying images. This case report did not require any institutional review board approval. 


\section{Funding}

This research did not receive any specific grant from funding agencies in the public, commercial, or Not-forprofit sectors.

\section{Disclosure}

The authors declare no conflicts of interest.

\section{References}

1. Bohun CM, Potts JE, Casey BM, Sandor GG. A population-based study of cardiac malformations and outcomes associated with dextrocardia. Am J Cardiol. 2007;100(2):305-309. doi:10.1016/j. amjcard.2007.02.095

2. Gutgesell HP. Cardiac malposition and heterotaxy. Sci Pract Pediatr Cardiol. 1998;1280:1539-1561.

3. Garg N, Agarwal BL, Modi N, Radhakrishnan S, Sinha N. Dextrocardia: an analysis of cardiac structures in 125 patients. Int $J$ Cardiol. 2003;88(2):143-155. doi:10.1016/S0167-5273(02) 00539-9

4. Vanpraagh R, Vanpraagh S, Vlad P, Keith JD. Anatomic types of congenital dextrocardia: diagnostic and embryologic implications. Am J Cardiol. 1964;13:510-531. doi:10.1016/0002-9149(64)90159-6
5. Van Praagh R. The importance of segmental situs in the diagnosis of congenital heart disease. Semin Roentgenol. 1985;20(3):254-271. doi:10.1016/0037-198X(85)90009-4

6. Applegate KE, Goske MJ, Pierce G, Murphy D. Situs revisited: imaging of the heterotaxy syndrome. RadioGraphics. 1999;19 (4):837-852. doi:10.1148/radiographics.19.4.g99j131837

7. Shaher RM, Duckworth JW, Khoury GH, Moes CAF. The significance of the atrial situs in the diagnosis of positional anomalies of the heart: I. Anatomic and embryologic considerations. Am Heart J. 1967;73(1):32-40. doi:10.1016/0002-8703(67)90306-7

8. Ellis K, Fleming RJ, Griffiths SP, Jameson AG. New concepts in dextrocardia. Am J Roentgenol. 1966;97(2):295-313. doi:10.2214/ ajr.97.2.295

9. Elliott LP, Jue KL, Amplatz K. A roentgen classification of cardiac malpositions. Invest Radiol. 1966;1(1):17-28. doi:10.1097/ 00004424-196601000-00025

10. Buxton AE, Morganroth J, Josephson ME, Perloff JK, Shelburne JC. Isolated dextroversion of the heart with asymmetric septal hypertrophy. Am Heart J. 1976;92(6):785-790. doi:10.1016/S00028703(76)80017-8

11. Mehrotra P, Choi JW, Flaherty J, Davidson CJ. Percutaneous coronary intervention in a patient with cardiac dextroversion. Bayl Univ Med Cent Proc. 2006;19(3):226-228. doi:10.1080/0899828 0.2006 .11928168

\section{Publish your work in this journal}

The International Medical Case Reports Journal is an international, peer-reviewed open-access journal publishing original case reports from all medical specialties. Previously unpublished medical posters are also accepted relating to any area of clinical or preclinical science. Submissions should not normally exceed 2,000 words or 4 published pages including figures, diagrams and references. The manuscript management system is completely online and includes a very quick and fair peer-review system, which is all easy to use. Visit http://www.dovepress.com/testimonials.php to read real quotes from published authors. 